\title{
Initial concepts for CELT adaptive optics
}

Richard G. Dekany, Brian J. Bauman, Donald T. Gavel, Mitchell Troy, Bruce A. Macintosh, et al.

Richard G. Dekany, Brian J. Bauman, Donald T. Gavel, Mitchell Troy, Bruce A. Macintosh, Matthew C. Britton, "Initial concepts for CELT adaptive optics," Proc. SPIE 4839, Adaptive Optical System Technologies II, (7 February 2003); doi: 10.1117/12.459798

SPIE Event: Astronomical Telescopes and Instrumentation, 2002, Waikoloa, Hawai'i, United States 


\title{
Initial concepts for CELT adaptive optics
}

\author{
Richard Dekany ${ }^{1}$, Brian Bauman ${ }^{2}$, Don Gavel ${ }^{2}$, \\ Mitchell Troy ${ }^{3}$, Bruce Macintosh ${ }^{2}$, Matthew Britton ${ }^{1}$ \\ ${ }^{1}$ Caltech Optical Observatories, California Institute of Technology, \\ Pasadena, CA 91125 \\ 2 Lawrence Livermore National Laboratory, University of California, \\ Livermore, CA 94550 \\ 3 Jet Propulsion Laboratory, California Institute of Technology, \\ Pasadena, CA 91109
}

\begin{abstract}
The California Extremely Large Telescope (CELT) project has recently completed a 12-month conceptual design phase that has investigated major technology challenges in a number of Observatory subsystems, including adaptive optics (AO). The goal of this effort was not to adopt one or more specific AO architectures. Rather, it was to investigate the feasibility of adaptive optics correction of a 30-meter diameter telescope and to suggest realistic cost ceilings for various adaptive optics capabilities. We present here the key design issues uncovered during conceptual design and present two non-exclusive 'baseline' adaptive optics concepts that are expected to be further developed during the following preliminary design phase. Further analysis, detailed engineering trade studies, and certain laboratory and telescope experiments must be performed, and key component technology prototypes demonstrated, prior to adopting one or more adaptive optics systems architectures for realization.
\end{abstract}

Keywords: Extremely large telescopes, multiconjugate adaptive optics, extreme adaptive optics

\section{INTRODUCTION}

The California Extremely Large Telescope (CELT) is a recently proposed ${ }^{1}$ visible and near-infrared telescope facility optimized for wide-field seeing limited observations and narrow-field diffraction-limited observations. The key structural design feature of this concept is a highly segmented, 30-meter diameter primary mirror designed to incorporate technical and economic lessons learned from the construction and operational experience of the twin Keck Telescopes on Mauna Kea, Hawaii.

During 2000-2001, the current CELT partners, California Institute of Technology and University of California, undertook a joint conceptual design study to explore the technical feasibility of building and operating a $30 \mathrm{~m}$ class astronomical observatory. The conceptual design was organized into working groups formed along lines of observatory subsystem, with significant personnel overlap between key subsystems. The working groups were: Adaptive Optics, Enclosure, Instrumentation, Science, Site, Software, and Telescope. The superset of the working groups and the engineering consultant and other parties of interest constituted a Design Team that conducted the work of the Conceptual Design Phase under the leadership of Prof. Jerry Nelson of University of California, Santa Cruz.

The Adaptive Optics Working Group consisted of Gary Chanan (UCI), Richard Dekany (CIT, Chair), Jerry Nelson (UCSC), and Andreas Quirrenbach (UCSD). The Design Team also included Brian Bauman (LLNL), Matthew Britton (CIT), Don Gavel (LLNL), Bruce Macintosh (LLNL), and Mitchell Troy (JPL). 


\section{STUDY CONSTRAINTS}

Based upon the scientific opportunities identified by the CELT Science Working group, the Design Team sought to balance the quality and quantity of science return from one or more AO capabilities. Constrained by the 12month duration of the study, and by certain interactions with other Observatory subsystems, we worked iteratively to identify promising regions of performance phase-space (i.e. residual wavefront error, field of view, throughput) for further study. The major constraints imposed by the telescope concept were the choice and location of telescope foci, the diameter of the telescope secondary mirror, and a desire to limit the cost estimate of the adaptive optics capabilities to $\sim 15 \%$ of the initial capital costs (with a strong endorsement for reserving a portion of the ongoing operations budget for future improvements). The justification for this approach was that AO technology is expected to be more easily upgraded than, for example, the diameter of the primary mirror or mechanical stiffness of the structure ${ }^{2}$.

In order to enable concrete comparisons, we selected a vertical turbulence model upon the Mauna Kea atmospheric model published by Roddier, et. al. ${ }^{3}$, with the following modifications:

$\begin{array}{llll}\text { Wavelength of parameter definitions } & \lambda & 500 & \mathrm{~nm} \\ \text { Coherence cell size } & \mathrm{r}_{0} & 20 & \mathrm{~cm} \\ \text { Greenwood frequency } & \mathrm{f}_{\mathrm{G}} & 50 & \mathrm{~Hz} \\ \text { Outer scale } & \mathrm{L}_{0} & >30 & \mathrm{~m} \\ \text { Isoplanatic angle } & \theta_{0} & 3 & \operatorname{arcsec} \\ \text { Maximum off-zenith science angle }^{4} & \zeta_{\max } & 65 & \mathrm{deg}\end{array}$

Table 1. Atmospheric parameters adopted for the conceptual design study.

Similarly we adopted as models for the distribution of natural guide stars the Bahcall-Soneira ${ }^{5}$ model for visible wavelengths and a similar distribution model by Wainscoat 6 for near-infrared wavelengths.

\section{MULTICONJUGATE ADAPTIVE OPTICS}

Multiple deformable mirrors, optically conjugated to different altitudes in the atmosphere, can increase the effective isoplanatic angle for $\mathrm{AO}^{7,8,9}$. Because of our scientific desire for a stable point spread function over a large field of view, we consider such a system for CELT. Furthermore, because some guide stars may be outside the classical isoplanatic angle (and would thus need to be operated in a non-null seeking mode), we expected overall improved performance with a multiconjugate system. Instrumentally, we envision this large field of view patrolled by a multiobject deployable integral-field spectrograph, or shared with multiple instruments.

Design trades in such a multiconjugate adaptive optics system can be made using error budget analysis by including the classical adaptive optics error terms (see, for example, reference ${ }^{10}$ ), along with new error terms that are specific to the use of multiple guide stars, and by modifying the error terms associated with deformable mirror errors, such as fitting error and generalized anisoplanatism. For a discussion of the various error terms in the CELT MCAO error budget, and their scaling dependencies, see the paper by Gavel, et. al., in this proceedings ${ }^{11}$.

\section{$\underline{\text { MCAO Error Budgets }}$}

Three different distributions of the error budget at different performance levels are presented in Table 2. It can be seen that the dominant error term for our model atmosphere is generalized anisoplanatism ${ }^{12}$, where in order to balance each performance level individually, we assumed the use of 2,3, and 4 deformable mirror conjugates for the case of $248 \mathrm{~nm}, 180 \mathrm{~nm}$, and $133 \mathrm{~nm}$ total $\mathrm{rms}$ residual wavefront error levels, respectively. It is worth noting that aliasing error, which arises in a Shack-Hartmann wavefront sensor ${ }^{13}$, and measurement errors due to laser guide star asterism deformations were omitted from this work due to the finite period of the conceptual study. We intend to address these 
terms in detail during the next phase of CELT. The major component technology levels needed to meet these example error budgets is given in Table 3 .

The calculation of the total number of actuators, as well as the distribution of actuators to different deformable mirrors, was made by minimizing the marginal utility of deploying the next actuator to a given deformable mirror ${ }^{14}$.

The calculation for the number of sodium laser guide stars was made invoking the premise that we wish to utilize existing, or nearly existing, sodium laser guide star technologies and not demand radically new performance levels. As a number of interesting laser technologies (e.g. fiber, vcsel, and color center lasers) are developing rapidly, this is perhaps a too conservative assumption but appropriate for our purpose of establishing MCAO feasibility for CELT. We use the tomography error terms calculated by Tokovinin and Viard ${ }^{15}$ to calculate the number of beacons needed in the LGS asterism. For measurement error, we take the routinely measured sodium light return from the Lick AO system wavefront sensor, 180 photons/subap/frame, and from this establish a baseline broadcast laser power of $11 \mathrm{~W}$ each, assuming the most favorable pulse format, the micropulse/macropulse format of the laser designed by Jeys ${ }^{16}$.

\begin{tabular}{|c|c|c|c|}
\hline \multirow{2}{*}{ Error term } & \multicolumn{3}{|c|}{ Total acceptable residual wavefront error } \\
\cline { 2 - 4 } & $248 \mathrm{~nm}$ & $180 \mathrm{~nm}$ & $133 \mathrm{~nm}$ \\
\hline Generalized anisoplanatism & 181 & 116 & 86 \\
\hline Fitting & 110 & 77 & 60 \\
\hline Residual primary figure & 50 & 50 & 50 \\
\hline Measurement & 80 & 70 & 40 \\
\hline Tomography & 51 & 39 & 32 \\
\hline Bandwidth & 50 & 35 & 25 \\
\hline Residual internal & 20 & 15 & 10 \\
\hline Residual instrument $^{\mathbf{1}}$ & 20 & 15 & 10 \\
\hline Equiv. Tip/tilt bandwidth $^{\mathrm{i}}$ & 20 & 15 & 10 \\
\hline Equiv. Tip/tilt anisoplanatism & 26 & 26 & 26 \\
\hline Equiv. Tip/tilt measurement & $\underline{12}$ & $\underline{12}$ & $\underline{6}$ \\
\hline Total RMS & 248 & 180 & 133 \\
\hline
\end{tabular}

Table 2. Example error budgets for three different rms residual wavefront error targets.

Importantly, for these calculations we have additionally assumed an LGS spot diameter of 0.4 " FWHM assuming the effects of LGS elongation for off-axis subapertures has been adequately addressed through either dynamic refocusing (pulse tracking) of the spot (which, admittedly, is not consistent with the pulse length of any existing sodium

${ }^{\mathrm{i}}$ Note that in Table 1, the contribution to the rms wavefront error for tip/tilt is usually handled separately as a contribution to Strehl ratio degradation given by,

$$
S R=\frac{1}{1+\frac{\pi^{2}}{2}\left(\frac{\theta}{\lambda / D}\right)^{2}}
$$

where theta is the rms image motion due to tilt. For the purpose of have a consistent tool by which to trade off the error budget terms, we choose to derive from Equation (1) an equivalent rms wavefront error for each tip/tilt error term, as if the observation were being conducted at $1 \mu \mathrm{m}$ wavelength, using the approximation,

$$
\sigma_{\text {equiv }}[n m]=\sqrt{-\ln (S R)} * \frac{1000}{2 \pi} \text {. }
$$

This allows us to rapidly explore the design parameter space using convenient simple linear optimizers, after which detailed effects of tip/tilt on image quality can be revisited. 
laser) or through synchronized WFS CCD clocking, assuming a custom radial geometry design of pixel locations on the wafer ${ }^{17}$. Another option for addressing LGS elongation is to cross two elongated LGS at each asterism location and combine the narrow and elongated spot motion measurements in an optimized fashion ${ }^{18}$. For the case of $133 \mathrm{~nm}$ wavefront error, we additionally assume it to be cost effective to correct the uplink beam of each LGS with it's own dedicated (low-order) AO system. Such uplink correctors are assumed to be diffraction limited on $50 \mathrm{~cm}$ laser projector apertures at $589 \mathrm{~nm}$ wavelength, and may therefore require Rayleigh laser beacons as guide stars for the uplink correction. The uplink AO systems need not reflect the CELT MCAO system architecture as they reside in a different region of AO parameter space. Low-cost curvature sensor AO systems are one uplink concept for future study.

\begin{tabular}{|c|c|c|c|}
\hline \multirow{2}{*}{ Component technology } & \multicolumn{3}{|c|}{ Total acceptable residual wavefront error } \\
\cline { 2 - 4 } & $248 \mathrm{~nm}$ & $180 \mathrm{~nm}$ & $133 \mathrm{~nm}$ \\
\hline Number of deformable mirrors & 2 & 3 & 4 \\
\hline Total number of deformable mirror actuators & 2,500 & 9,000 & 20,600 \\
\hline Number of sodium laser guide stars & $5-7$ & $7-9$ & $9+$ uplink AO \\
\hline
\end{tabular}

Table 3. Example technology levels for three different rms residual wavefront error targets.

\section{Design Example for a 4-DM, SH WFS System}

To further explore the details with an example, we shall restrict our discussion to an MCAO system capable of meeting the $133 \mathrm{~nm}$ wavefront error level over a 1' field of view (FoV) (which, incidentally, provides $292 \mathrm{~nm} \mathrm{rms}$ wavefront error over a 2' FoV). The parameters describing this system are presented in Table 4.

\begin{tabular}{|c|c|}
\hline \multicolumn{2}{|c|}{ MCAO illustrative design } \\
\hline Number of deformable mirrors & 4 \\
\hline Conjugate layer heights & $0,3.0,5.8$, and $12.0 \mathrm{~km}$ \\
\hline Number of DM actuators (inscribed) & $7700,7000,4200,1780$ \\
\hline Total number of DM actuators & 20600 \\
\hline Wavefront sensor subaperture diameter & $32 \mathrm{~cm}$, projected onto sky \\
\hline Number of sodium laser guide stars & 9 \\
\hline Brightness of laser guide star & 180 photodetections/subaperture/frame \\
\hline Number of natural guide stars & Between 3 and 9, optimum unknown \\
\hline Brightness of primary tip/tilt guide star & $\mathrm{m}_{\mathrm{H}}=15.1$ \\
\hline Brightness of auxiliary tip/tilt guide stars & $\mathrm{m}_{\mathrm{H}}<23$ \\
\hline
\end{tabular}

Table 4. Illustrative MCAO design parameters that meet the $133 \mathrm{~nm}$ total error budget presented in Table 2 .

\section{$\underline{\text { MCAO Relay Optics }}$}

The example 4-deformable mirror MCAO optical relay consists of a pair of 1:1 conjugate off-axis parabola (OAP) relays. Each OAP pair can individually correct spherical aberration and coma perfectly, but is limited by residual astigmatism. By using two OAP relays, for a total of 4 identical OAP mirrors, astigmatism can also be corrected. Details of this optical design are presented elsewhere ${ }^{19}$.

The relationship of the MCAO relay to the telescope and instruments is shown in Figure 1. The large disk in this figure represents the $30 \mathrm{~m}$ diameter primary mirror of CELT. The MCAO system is shown here on the left-hand $30 \mathrm{~m} \times 15 \mathrm{~m}$ Nasmyth platform. The platform is shown in this view as partially transparent in order to reveal an instrument oriented as a vertical rotating drum (VRD), which enables correction of field rotational with minimal mechanical flexure. 


\section{Image and Pupil Rotation}

CELT is conceived as an altitude-azimuth mount telescope ${ }^{20}$. As a result, the image of the sky at the Nasmyth focal plane rotates with respect to instrumentation fixed on the platform. Furthermore, the relayed pupil and the field both rotate under the influence of the articulated tertiary mirror that feeds the Nasmyth instruments. We assume for now that field rotation will be taken out by rotating the science instruments, and that field and pupil rotation will be compensated in the MCAO system by rotating the entire WFS (as a vertical rotating drum, or VRD), and simultaneously rotating each of the deformable mirrors. Although this approach has better throughput than any solution exploiting a " $\mathrm{K}$ " mirror, this preliminary choice has other complications and will be revisited in detail during the CELT preliminary design phase.

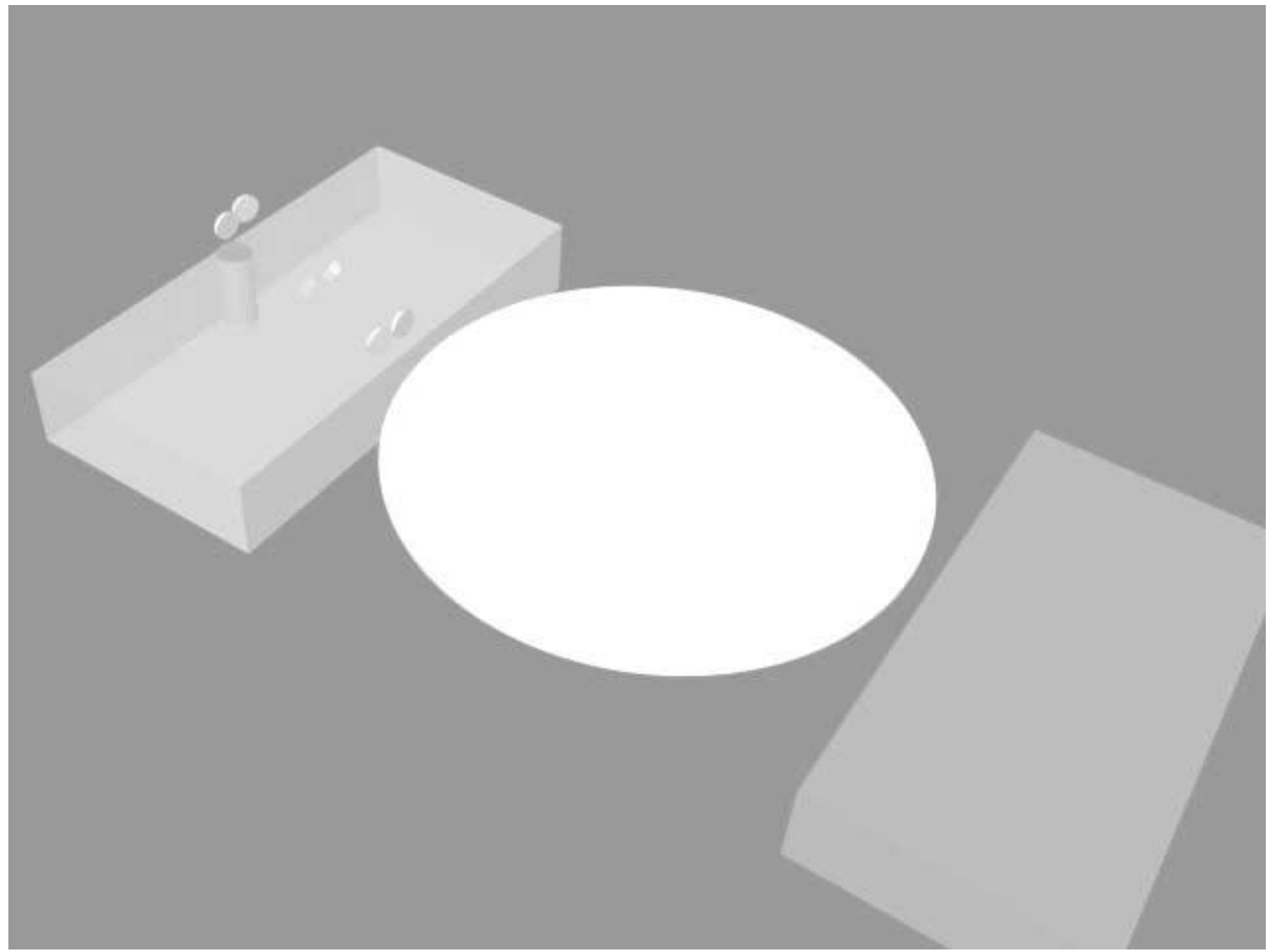

Figure 1. Schematic drawing of the MCAO double OAP relay residing on the left-hand Nasmyth platform of the 30m diameter primary mirror CELT telescope. The relay consists of 4 OAPs which form two collimated spaces, each containing two of the deformable mirror conjugates. The relay feeds a vertical rotating drum (VRD) instrument shown beneath the MCAO system.

The image derotation requirements are more stringent than for the $10 \mathrm{~m}$ Keck Telescopes. Here, we assume it is desirable to keep blurring due to imperfect image derotation to less than one-tenth of the point spread function width. For seeing limited observations, over a 20 arcmin FoV, a goal of less than $0.5 / 10=0.05$ arcsecond requires a rotation angular error of less than $17.5 \operatorname{arcseconds}^{21}$. For MCAO, over a $1 \mathrm{arcmin}$ FoV, a goal of less than $0.007 / 10=0.0007$ arcseconds requires a rotation angular error less than 4.9 arcseconds. 


\section{Laser Asterism Rotation}

If LGS are projected from locations fixed on the perimeter of the primary mirror (for example), the orientation of the asterism on the sky will change as the telescope tracks an astronomical target across the sky. The rate of asterism rotation in this model is linked to the pupil rotation rate, which differs from the natural field rotation rate. For this reason (among others), the Keck strategy of field derotation through use of a "K" mirror before the AO system cannot simultaneously stabilize the instrument and WFS focal planes. It therefore becomes favorable to consider instrument rotation as stabilizing the science target, while rotation of the WFS is used to stabilize the LGS asterism relative to the sensor. (Although the CELT instruments will be large, if their rotation axes are oriented along the gravity vector, the mechanical flexure is likely to be minimized and the instrument rotator can be independently designed (e.g. the instrument can be place on a rotating table in the 'basement' of the Nasmyth platform.)

\section{EMMISSIVITY MINIMIZED ADAPTIVE OPTICS}

The large diameter of the CELT telescopes provides an exceptional opportunity for high-resolution midinfrared observations (the diffraction limited resolution at ten micron wavelength is $\lambda / D=69$ milliarcseconds.) Although cryogenic space astronomical missions, such as NGST, can surpass CELT in terms of raw point source sensitivity, the resolution advantage of CELT warrants a consideration of adaptive optics optimized for minimum thermal emission. Fortunately, the density and brightness of natural guide stars alone appears sufficient for most mid-IR observations with good sky coverage (down to perhaps $5 \mu$ wavelength). Furthermore, the isoplanatic angle at mid-IR wavelengths is sufficient for single-conjugate correction over our adopted $30 \operatorname{arcsec}$ scientific FoV. Thus, in many ways the complexity of a mid-infrared $\mathrm{AO}$ system for a 30-meter telescope is significantly simpler than that of the existing $\mathrm{AO}$ systems at Keck Observatory.

\section{Design Options}

There are two obvious approaches to minimizing the emissivity of a low-order, single-conjugate adaptive optics system, either minimize the number of emitting optical elements or minimize the emission of the optical elements. In practice, this choice can be realized as either implementing an adaptive secondary mirror AO system, or by significantly cooling a more conventional AO system, perhaps to $77 \mathrm{~K}$. The ultimate combination of the two principals has not been previously explored in detail, namely the use of a cryogenic AO system at prime focus, where the number of warm surfaces is reduced to only the primary mirror, and the input window of the cryostat. Additional factors, such as which focus is used affect the design as summarized in the following table.

\begin{tabular}{|c|c|c|c|}
\hline \multirow{2}{*}{ Concept } & \multicolumn{3}{|c|}{ Number of warm surfaces (including dewar window) } \\
\cline { 2 - 4 } & Prime focus* & Cass focus* & Nasmyth focus \\
\hline Adaptive secondary & -- & 3 & 4 \\
\hline Cryo-AO & 2 & 3 & 4 \\
\hline 'Warm' AO & $\begin{array}{c}3 \\
\text { (no implemented } \\
\text { systems) }\end{array}$ & $>4$ (typ. 11) & $>5$ (typ. 12) \\
\hline
\end{tabular}

Table 5. Options for emissivity minimization for mid-infrared observations with CELT. * The current CELT concept does not include either prime or Cassegrain focal stations.

\section{$\underline{\text { Sensitivity Comparison }}$}

To help evaluate the relative advantage and disadvantage of these designs, we applied the analytical technique (and generously shared software) from Lloyd-Hart ${ }^{22}$, to the CELT situation. The metric for comparison is the relative integration time required to reach the same signal-to-noise ratio that could be obtained from a cryogenic prime focus AO system. Our assumptions for atmospheric emissivity (which is dominated by a conservative choice of precipitable water vapor), and for the quality of our mirror coatings (operating in the field) are shown in Table 6. 


$\begin{array}{ll}\text { Air temperature } & 275 \mathrm{~K} \\ \text { Precipitable water vapor } & 3 \mathrm{~mm} \\ \text { Primary mirror reflectivity } & 95 \% \\ \text { Secondary mirror reflectivity } & 97 \% \\ \text { Reflectivity of subsequent mirrors } & 98 \% \text { each } \\ \text { Transmission of subsequent windows } & 98 \% \text { each }\end{array}$

Table 6. Emissivity model assumptions.

The resultant comparison of the relative integration times, as integrated across the usual infrared observing bands, is shown in Table 7.

Average ratio of integration time to achieve the same

SNR relative to cryogenic prime focus AO system

$\begin{array}{lcc}\text { Wavelength Band } & \begin{array}{l}\text { Cryo-Nasmyth or } \\ \text { adaptive secondary Nasmyth system } \\ \text { (4 warm surfaces) }\end{array} & \begin{array}{l}\text { Warm MCAO } \\ \text { system at Nasmyth } \\ \text { (14 warm surfaces) }\end{array} \\ \text { K }(2.2 \mu \mathrm{m}) & 1.32 & 3.31 \\ \text { L }(3.4 \mu \mathrm{m}) & 1.51 & 4.76 \\ \text { M }(5.0 \mu \mathrm{m}) & 1.34 & 3.51 \\ \text { N }(10.6 \mu \mathrm{m}) & 1.35 & 5.41\end{array}$

Table 7. Relative integration time to detect a background-limited point source to the same SNR (for several emissivity optimization options) relative to the integration time for the ultimate optimization, a cryogenic prime focus system.

The relative integration time is a strong function of the atmospheric background, but it is apparent that the penalty of either an adaptive secondary system or a cryogenic AO system at Nasmyth is small compared to the dramatic increases in integration time necessary with a warm $(0 \mathrm{C}) \mathrm{MCAO}$ system at Nasmyth. It is interesting to note that the integration time multiplier of the Cryo-Nasmyth system over an adaptive secondary Cassegrain system (3 warm surfaces) is not sufficient in our opinion to warrant a Cassegrain focus station, which would be a major structural complication.

\section{Cryo-prime Focus AO}

To demonstrate the optical feasibility of a prime focus adaptive optics system, we designed a simple relay, based upon a one-meter class ellipsoidal deformable mirror, shown in Figure 2. This design provides a well corrected $30 \mathrm{arcsec}$ FoV by taking advantage of an interesting feature of the CELT primary mirror, namely its high segmentation and large actuator stroke $(2 \mathrm{~mm})$ for each segment (the stroke requirement is driven by gravity deflections of the primary mirror). It is possible to simplify the prime relay design by actively driving the conic constant of the primary from its nominal hyperbolic configuration through parabolic and into an ellipsoidal configuration. The magnitude of the high spatial frequency aberrations induced by using individual segments optimized as a hyperboloid as an ellipsoid is of order $120 \mathrm{~nm} \mathrm{rms}{ }^{23}$. The optical performance of this relay is excellent over 30 " FoV for observing wavelengths as short as 3.5 microns.

The development of large cryogenic aspheric, though low-order, DM is a significant technology challenge, but not in our opinion any more difficult than the alternative approach of scaling the existing mirror technology of the Arcetri/Arizona group to CELT-class adaptive secondaries. For a hypothetical telescope primary having a faster F-ratio, the technical challenge of adaptive secondaries is decreased. If wind-induced wavefront errors are found to require greater amplitude of control than currently anticipated, the high-stroke technology of the Arcetri/Arizona group may 
become desirable, even if the secondary did not possess sufficient actuator count or bandwidth to act as the only deformable mirror at its conjugate plane.

\section{$\underline{\text { LOAO Error Budgets }}$}

Mid-infrared observing wavelengths have the advantageous property that they can suffer considerable residual wavefront distortions without significant degradation of the image quality. We have chosen to use $500 \mathrm{~nm}$ residual rms wavefront error as a tolerance for the development of engineering error budgets, as shown in Table 8.

We assume wavefront sensing for this mode utilizes near-infrared photons, to both maximize the sensing signal and to avoid the potential errors due to differing atmospheric paths taken by visible light and mid-IR light due to dispersion. From Table 8, we see that the combination of anisoplanatism and measurement error dominate the error budget, which is a consequence of wanting the LOAO mode to employ only natural guide stars, over a significant fraction of sky. In many case of real observation, anisoplanatism and measurement error will both be less than shown in these error budgets, allowing good correction as short as 3.5 microns. When high sky coverage fraction near the galactic pole and 3.5 micron observing wavelength are both required, it is envisioned that these observations will be made with the MCAO system, with a correspondingly higher infrared background level due to the MCAO system's many emissive surfaces.

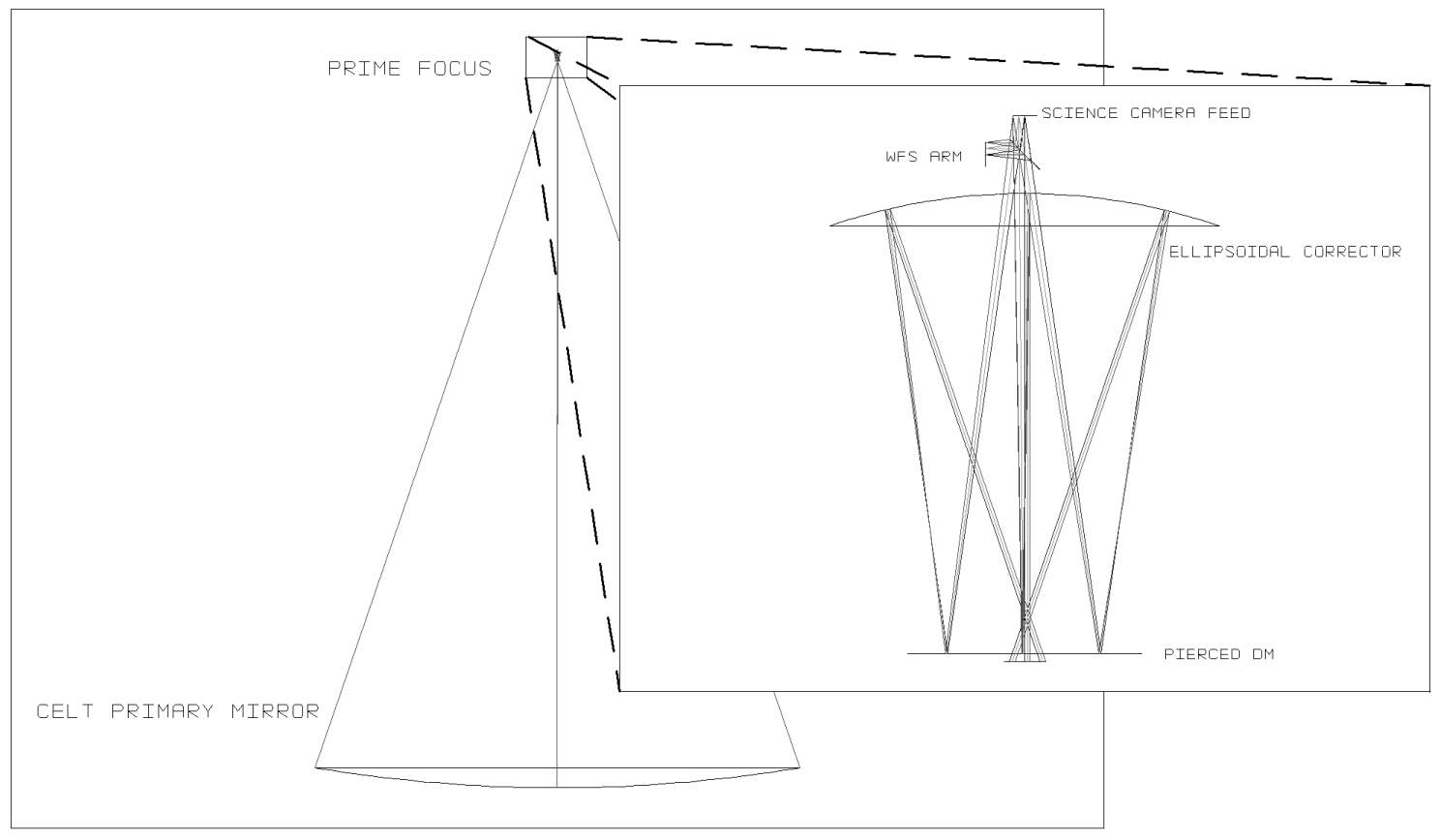

Figure 2. Schematic of ellipsoidal-DM relay for a prime focus adaptive optics system. Details of the wavefront sensor, science instrument, and the field derotator are not shown.

\section{Nodding, Chopping, Counternodding, Counterchopping}

Although time constraints allowed our group little time to consider detailed strategies for mid-IR calibration, it is believed that modest speed $(2 \mathrm{~Hz})$ chopping will be possible with telescope secondary mirror ${ }^{24}$. The determination of whether the LOAO system fast control loops would remain locked during chopping and nodding (which would require active counter-chopping or counter-nodding) remains to be investigated. 


\begin{tabular}{|c|c|c|c|c|}
\hline \multirow{3}{*}{$\begin{array}{c}\text { Error term } \\
\text { Anisoplanatism } \\
\text { (15" to guide star) }\end{array}$} & \multicolumn{4}{|c|}{ RMS wavefront error [nm] } \\
\hline & \multicolumn{2}{|c|}{$\theta_{0}[0.5 \mu]=3 \operatorname{arcsec}$} & \multicolumn{2}{|c|}{$\theta_{0}[0.5 \mu]=2 \operatorname{arcsec}$} \\
\hline & & 225 & & 319 \\
\hline Measurement & $m_{K}=16.1$ & 270 & $\mathrm{~m}_{\mathrm{K}}=14.8$ & 226 \\
\hline Bandwidth & $(5.3 \mathrm{~Hz},-3 \mathrm{db})$ & 238 & $(6.7 \mathrm{~Hz},-3 \mathrm{db})$ & 195 \\
\hline Fitting & (400 actuators) & 193 & & 193 \\
\hline $\begin{array}{c}\text { Non-correctable telescope } \\
\text { figure errors }\end{array}$ & & 75 & & 75 \\
\hline $\begin{array}{l}\text { Non-correctable internal } \mathrm{AO} \\
\text { figure errors }\end{array}$ & & 40 & & 40 \\
\hline $\begin{array}{l}\text { Non-correctable instrument } \\
\text { figure errors }\end{array}$ & & 40 & & 40 \\
\hline $\begin{array}{l}\text { Equivalent tip/tilt } \\
\text { measurement }\end{array}$ & $\begin{array}{l}\text { (using high-order } \\
\text { guide star) }\end{array}$ & 106 & & 70 \\
\hline $\begin{array}{c}\text { Equivalent } \\
\text { tip/tilt bandwidth }\end{array}$ & $(6.3 \mathrm{~Hz},-3 \mathrm{db})$ & 74 & $(8.4 \mathrm{~Hz},-3 \mathrm{db})$ & 49 \\
\hline $\begin{array}{l}\text { Equivalent tip/tilt } \\
\text { anisoplanatism }\end{array}$ & $\begin{array}{l}\text { (using high-order } \\
\text { guide star) }\end{array}$ & 83 & & 75 \\
\hline Total wavefront error & & 500 & & 500 \\
\hline
\end{tabular}

Table 8. Example error budgets for a mid-infrared, emissivity optimized AO capability, for two different isoplanatic angles. In both cases, $\mathrm{r}_{0}[0.5 \mu]=0.20$ meters is assumed.

\section{REFERENCES}

1 “California Extremely Large Telescope Conceptual Design for a Thirty-Meter Telescope," prepared and published privately by California Institute of Technology and University of California, June 2002.

${ }^{2}$ The counterargument that observatories have historically found it very challenging to fund ongoing instrument programs is expected to be addressed separately.

${ }^{3}$ Roddier, F., Cowie, L., Graves, J., Songaila, A., McKenna, D., "Seeing at Mauna Kea - A joint UH-UN-NOAO-CFHT study," Proc SPIE, 1236, pp. 485-491 (1990).

${ }^{4}$ Although we allow for a maximum zenith angle of 65 degrees, all subsequent calculations presented here assume viewing at zenith, unless otherwise noted.

5 Bahcall \& Soneira, "The Universe at faint magnitudes, I. Models for the Galaxy and the predicted star counts," AstrophysJ. Suppl., 44, pp. 73-110 (1980).

${ }^{6}$ Wainscoat, R. J., et al., "A model of the 8-25 micron point source infrared sky," Ap. J. Suppl, 83, pp. 111-146, (1992).

${ }^{7}$ Beckers, J. M, Proc. SPIE, 1114, 215, (1988).

${ }^{8}$ Tallon and Foy, A\&A 235, 549 (1990).

${ }^{9}$ Ragazzoni, Marchetti, and Rigaut, A\&A 342, L53 (1999).

${ }^{10}$ Hardy, J. W., Adaptive Optics for Astronomical Telescopes, Oxford University Press (1998).

${ }^{11}$ Gavel, Bauman, and Dekany, "Scaling laws for designing multi-conjugate multi-guidestar adaptive optics", Proc. SPIE, 4839, (2002).

${ }^{12}$ Tokovinin and Le Louarn, "Isoplanatism in a mult-conjugate adaptive optics system," JOSA A 17, 1819-1827, (2000).

${ }^{13}$ Rigaut, Veran, and Lai, Proc SPIE, 3353, 1038 (1998).

${ }^{14}$ Dekany, R., Gavel, D., and Bauman, B, private communications.

${ }^{15}$ Tokovinin and Viard, JOSA A, 18, 873-881 (2001).

${ }^{16}$ Jeys, Brailove, and Mooradian, "Sum frequency generation of sodium resonance radiation," App. Optics 28, 2588-91, (1991).

${ }^{17} \mathrm{~J}$. Beletic of Keck Observatory, private communication, has suggest such a custom CCD design may be quite feasible. 
${ }^{18}$ It has been estimated that the total increase in laser power via this technique is only a factor of two greater than if no LGS elongation were present on CELT, J. Nelson, private communication.

${ }^{19}$ Bauman and Dekany, "Multiple conjugate adaptive optics (MCAO) system for extremely large telescopes", Proc. SPIE 4839, (2002).

${ }^{20}$ Wilson, R. N., Reflecting Telescope Optics, Vol. I and II, Springer, New York, (1999).

${ }^{21}$ Nelson, J., Keck Observatory Report No. 49, University of California, (1981).

${ }^{22}$ Lloyd-Hart, M., et. al., "Infrared adaptive optics system for the 6.5-m MMT: system status and prototype results", Proc. SPIE, 3353, pp. 82-93, (1998).

${ }^{23}$ See Nelson, J., "How well will CELT approximate a parabola?", CELT Technical Note \#7, (2001).

${ }^{24} \mathrm{~J}$. Nelson, private communication. 